\title{
AUTOMATIC HUMAN HEART SEARCH ON CT-SCANS
}

\author{
Mariya Dubrovskaya \\ Lobachevsky State University of Nizhni Novgorod \\ Russian Federation, 23 Prospekt Gagarina (Gagarin Avenue)
}

\begin{abstract}
Here is proposed a solution for actual problem of heart allocation on tomography data. One of the most popular tomography types used for various heart investigations is CT, thus the technology proposed here is initially developed for CT data. The main part of the algorithm consists of four stages: preliminary source volume limitation, forming hypotheses of lungs location, determination of approximate surrounding heart cube and searching for a point within the cube which is for sure inside the heart. The algorithm also includes a preliminary stage: coordinate system allocation. This stage is essential when position of a scanned patient is unknown relative to a tomograph. During the stage we find, where are left and right, top and bottom, front and back sides of human body. The stage consists also of four parts itself: definition of $\mathrm{Ox}$ and $\mathrm{Oy}$ axes and determination of $\mathrm{Oy}, \mathrm{Oz}$ and $\mathrm{Ox}$ directions, by analyzing anatomy of a patient, with regard to standard human anatomy. The method is fast and doesn't require any human guidance and preliminary adjustment. The whole method is convenient as automatic preprocessing for unlimited scope of problems, e.g. segmentation, surface or solid model reconstruction, etc. The proposed method provides flexible way for further local heart positioning refinement and works even for static, not timed, tomography data.
\end{abstract}

\section{KEYWORDS}

Localization, Surrounding Cube, Hypothesis, Preprocessing, Approximation, Cardiac

\section{INTRODUCTION}

Almost every task of heart processing on tomography data starts with attempts to understand where the heart locates. Investigation problems, such as segmentation, surface reconstruction, etc. often allow to guide heart position for an algorithm manually. On the contrary, in real clinical applications it becomes more and more inconvenient and hard to make these actions every time. Therefore, it is useful to have a tool, able to find a target organ, heart, without user guidance.

Currency of the examined problem remains due to insufficient experience in automatic heart search. Investigated methods are mostly limited to requirement of time scale in data. There are attempts to use entropy of the intensity changes (Atehortua, 2016a) and center-surround principle (Atehortua, 2016b). Absolute difference between the first and the following frames (Fadil, 2017) is also computed. Furthermore, mean image and absolute error (Ma, 2016) were calculated. These inventors tried heart search on MR data, so that the methods require timed tomography, because they detect regions changing in time. Similar methodologies were invented by the following researchers. Background estimation and Laplacian pyramid (Hoffmann, 2016) were used, linear combination of time functions was researched with 3D labeling in order to ensure spatial continuity (Constantinides, 2010), simple technique of subtracting (Farag, 2012) was applied, differences between images (Sorgel, 1997) were computed together with automatically determined intensity threshold, standard deviation was used (Hadhoud, 2011) and optical flow (Saleh, 2016) was tried. These methods are based on MR data and the assumption that heart is the strongest moving structure in the CMR (Cardiovascular magnetic resonance imaging) data, hence they impose a requirement of time dimension presence. Big amount of methods for MR imaging and of their usages demonstrate their easiness and the fact that such methods are classical for MR. There is also a work with 3D data field (Lei, 2016), however time-domain characteristics of 3D data for heart ROI extraction are used there as well. Another research (Huang, 2007) is also based on spatio-temporal data and on motion and is tested on 4D MRI and tagged MRI images of heart. Heart region localization on chest X-rays (Candemir, 2016) was introduced. The author tried intensity histogram analysis in accordance with anatomy knowledge. The method uses existing 
chest X-rays and their manually marked heart boundaries as models, and estimates the heart boundary of a patient X-ray by registering the model X-rays to the patient X-ray. A heart localization method based on Computed Tomography Angiography (CTA) images and Generalized Hough Transformation (Saalbach, 2011) was proposed. The method implies templates and learning usage and sufficiently time-consuming execution. Automatic heart localization, based on machine learning (Zheng, 2008), was provided. A recursive ray casting mechanism for cardiac CTA scans (Kim, 2009) and thoracic CT data (Lorenz, 2005) was also used. The method is based on intensities analysis and anatomy knowledge. Unfortunately, recursion implies memory dependence. Motion fields in CT images (Tavakoli, 2013) were tried.

The method, proposed here, doesn't depend on time scale. The input of the method is CT data without any preprocessing, and the result is a point, that for sure belongs to heart (is positioned inside this organ).

\section{METHODOLOGY}

The whole method consists of the four main sequential stages $(2.2-2.5)$ and an auxiliary stage 2.1, explained in detail below. Here is presented the chronological order of all stages`start (Figure 1).

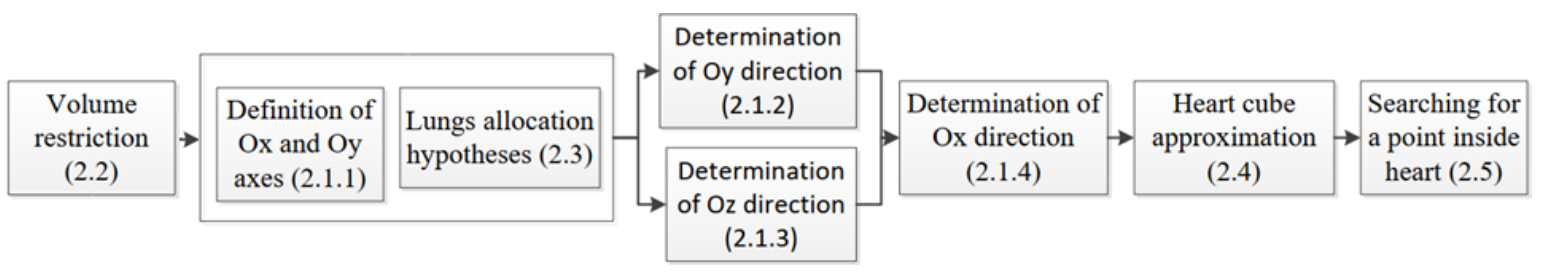

Figure 1. Methodology Sequence Diagram

\subsection{Axes Allocation}

The main heart search mechanism is preceded by establishment of accordance between orthogonal axes and the body sides. This step is essential for fully automatic algorithmic search and is automatic itself. Requirement of the stage is explained by the fact that we must navigate within CT-scans volume in order to find a desired object. In theory, a tomograph can scan a patient starting with any of eight angles of the scanned volume. Additionally, the patient can be disposed differently concerning the tomograph, that's why at first it is required to understand, where each side of the body locates. After that, it is possible to process further anatomical peculiarities of a human body for the search. The single assumption of algorithm declares that body lies along $\mathrm{Oz}$ axis. I define direction of $\mathrm{Oz}$, location of $\mathrm{Ox}$ and $\mathrm{Oy}$ axes and their directions.

\subsubsection{Definition of Ox and Oy Axes}

First of all, we need to define the rest of axes directions within Oxy plane. In fact, the stage 2.2 of the main search mechanism is applied before this step. Then executions of this (2.1.1) and 2.3 sections are combined. According to 2.1.1, the stage 2.3 is implemented twice, concerning two possible allocations of Ox and Oy axes within a slice. In order not to destruct primary logic, descriptions of stage 2.3 , as well as of 2.2, are retained in appropriate sections below, therefore it`s suggested to get acquainted with 2.2 and 2.3 first, and then continue reading from here. So, here I try to construct hypotheses of lungs along both axes within a slice. Distance inside a lung is relatively long along the direction between breast and spine in comparison with another orthogonal direction within Oxy. The axis, along which there are more hypotheses, is named as Oy. The latter means, that we dispose Oy axis along direction between breast and spine.

\subsubsection{Determination of Oy Direction}

Now, when all three axes are located, we need to define their directions. Let the direction of Oy be pointed from spine towards breast. Therefore, we need to distinguish both appropriate sides of the body (which contain accordingly spine and breast). In order to find this direction, the spine location is detected. Here results of 2.3 together with 2.1 .1 are used as well. The middle Oy coordinate of lungs hypotheses is used in 
order to subdivide an experimental slice of the chest onto two halfs, as only one such half can contain spine anatomically. Such a slice must contain chest, so that any of slices with successful lungs hypotheses can be chosen for this objective, e.g. the middle of them. Within each half of the slice, limited to the body cube, I move a little square frame of size comparative to spine`s 2D trace in Oxy. This shape of frame was chosen because spine trace in Oxy is similar to such symmetric shape as a square, for instance. For each half of slice I look for the frame with the biggest number of bone densities. Then I compare the maximum frames` counters of both halfs. The half with the biggest of maximums is declared as the side which contains spine.

\subsubsection{Determination of $\mathrm{Oz}$ Direction}

In this step the results of 2.3 together with 2.1.1 are also used. Let Oz be directed from legs towards head. Therefore, we need to understand, where legs and head are. I assume that both lungs' size in body in the direction between arms widens from lungs` highest part (nearest to head) towards the lowest (nearest to legs). Thus, I compare distances along Ox axis (between arms) in the hypotheses of the highest and lowest slices with successful lungs hypotheses. The one of them with the biggest distance is determined as nearest to legs.

\subsubsection{Determination of Ox Direction}

Now, when we know all three orthogonal axes` locations and directions of two of them, we can calculate direction of the latter. If you look attentively at the human body coordinate system, where $\mathrm{Oz}$ is directed along the body from legs towards head and Oy is directed from spine towards breast, you can see, that the vector from axes' center towards the left arm is located unambiguously with regard to two other axes, because they form a left-hand triple. I just let the direction towards the left arm be a positive direction of Ox (which means, that $\mathrm{Ox}$ is directed from the right arm towards the left). Using mixed product we easily determine the side of the left body half in CT volume.

\subsection{Volume Restriction}

This step is executed before 2.1.1 (read 2.1.1 for details). The main algorithm begins with source volume limitation for more efficient search. It doesn`t make sense to process the volume that is known not to contain the desired object. Thus, surrounding volume including only air-like substances, are excluded from consideration. It is the first stage of the main algorithm. In order to reach such result the algorithm runs along each of three axes from both endings and stops when it meets any density of either muscle or bone. Hounsfield unit (HU) nature allows such references to different tissues and densities of many objects, because the ranges of various substances are computed and known. All the six spaces between each CT volume`s border and an appropriate coordinate of stop are excluded. Hence, the volume for further processing includes whole scanned human body, so that the space that doesn`t include it for sure is ignored.

\subsection{Lungs Allocation Hypotheses}

This step is executed along with 2.1.1 (read 2.1.1 for details). Hypotheses of lungs allocation are formed at each slice of reduced volume. Algorithm casts parallel rays through data volume within Oxy and detects sufficiently long continuous distances of lungs densities, which lie between shorter, but thick enough, distances of body tissues densities. Note, that both lungs must present at CT-scans. Each hypothesis is represented as a couple of points that denote the start and the end of approximate lung position. Examples of hypotheses are demonstrated in the figure (Figure 2, a). When all hypotheses are found, their connectedness is checked up. There shouldn't be long intervals between hypotheses along Oz. Only that largest subset of hypotheses is chosen as successful, where all of them locate close to one another along Oz.
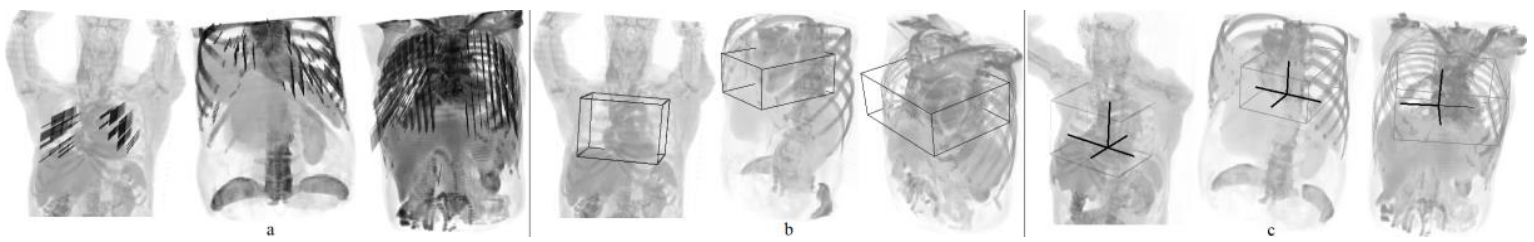

Figure 2. a) Hypotheses of hearts`allocations; b) Surrounding hearts`cubes; c) Determined points inside hearts 


\subsection{Heart Cube Approximation}

From slices, which contain hypotheses, those are chosen, where there are two hypotheses or more. Then only those are retained, that contain at least one couple of neighbor hypotheses, divided by long enough distance. The first such pair is chosen for each slice. Then extreme coordinates are chosen from successful hypotheses collected from all slices, along both horizontal orthographic axes: the least and the biggest along each axis. Extreme coordinates along vertical axis are defined below. All the limits are used for surrounding heart cube (to be precise, parallelepiped) to be built, where six coordinates are required, two for each axis. These coordinates are very approximate limits, containing heart inside. However, it is necessary to consider several anatomical peculiarities. Firstly, it is necessary to correct the surrounding box along direction between arms. We have to expand the left extreme border proportionally towards the left in order to include Apex for sure (all such proportions are described in section 2.6, identification between body sides and axes is described in 2.1). Note, that patient`s body mustn`t locate at CT-scans at significant angles with orthogonal axes (usually, it doesn `t). Secondly, both extreme coordinates along another horizontal direction (between spine and breast bone) should be proportionally enlarged towards breast, because heart is shifted towards breast bone with regard to lungs and spine. Thirdly, the middle coordinate along vertical axis is chosen from successful hypotheses, and then similar indents are added in both directions from this center. Sample surrounding hearts` cubes are shown in the figure (Figure 2, b) for several CT-scans.

\subsection{Searching for a Point inside Heart}

The algorithm searches for sufficiently long continuous distances, comparable to heart size (about it`s half or less; this proportion is also described in section 2.6), of such tissues as muscles, blood and blood contrast along all three orthogonal axes within found heart cube. Thus, as a result, here is detected some point, which for sure lies inside heart. After this step more precise and full characteristics of heart can be determined, for any purpose. By moving out of the point, it is possible to approximate position of the whole heart, build heart surface or solid heart model, etc. Such points inside hearts are denoted as intersections of three lines inside hearts` cubes in the figure (Figure 2, c) for some CT-scans.

\subsection{Experiments}

All parameters, used in the algorithm, are considered to satisfy every concrete patient body`s proportions. Formally, each of the parameters can be tuned in case of misses in proportions, however in this work I examine only the solution, that doesn`t include user`s manual postprocessing. General necessary body proportions were considered as mutual relations taken from several CT-scans. The relations turned out to be similar, but they still were averaged. All proportions have a single and simple base: surrounding body box. I took the following proportions: threshold for necessary (minimal) lungs length (lungs_len_thres) is: body cube dimension in the direction between arms (dim_arms) divided by 5; threshold for necessary heart size (among distances between rays) is: lungs_len_thres divided by 2 ; heart cube limit in the direction between head and legs, nearest to legs is: middle lungs` coordinate extended in the direction from head to legs by "lungs len thres divided by 4"; heart cube limit in the direction between head and legs, nearest to head is: middle lungs' coordinate extended in the direction from legs to head by "lungs_len_thres divided by 4"; both heart cube limits in the direction between breast and spine are: shift in the direction from spine towards breast by "(dim_arms divided by 25) and multiplied by 2"; heart cube limit in the direction between arms, nearest to the right is: the coordinate from hypotheses, nearest to the right arm (without additional adjustment); heart cube limit in the direction between arms, nearest to the left is: the coordinate from hypotheses, nearest to the left arm, extended in the direction from the right to the left by "dim_arms divided by 25"; the frame side for skeleton search is: dim_arms divided by 10 . The parameters were taken for processing of these and all other CT-scans. Given parameters don't require repeated adjustment, as they are based on standard anatomy, so they can be taken from here as ready constants. All iterations along rays should have sizes, that intersect all considerable tissues` passages. Intensities` thresholds for tissues and substances must conform with HU standards. 


\section{CONCLUSION}

The search method, presented here, results in a point, which belongs to heart or any of tissues inside heart. Additionally, approximate bounding box is built around heart, which is a bit wider than the heart itself. Outputs of all algorithm`s stages mostly agree with visual intuitive analogues, which could be implemented manually. The method is convenient as preliminary stage for any heart processing, such as surface or solid model reconstruction, etc. Instead of manual specification of heart location on CT-scans for each person and for each task, it is possible to set real heart position quickly using proposed method of automatic heart search.

The method is simple, fast (about a few seconds for a single full-body CT-scans on Intel ${ }^{\circledR}$ Core ${ }^{\mathrm{TM}} \mathrm{i} 7-7700 \mathrm{HQ} 2.80 \mathrm{GHz}$ processor), fully automatic and doesn't require human guidance, preliminary adjustment and time scale. This method is convenient as automatic preprocessing for unlimited scope of problems regarding human heart. Proposed method provides flexible way for further local heart positioning refinement.

\section{REFERENCES}

Atehortua, A. et al, 2016. Automatic segmentation of 4D cardiac MR images for extraction of ventricular chambers using a spatio-temporal approach. Medical Imaging 2016: Image Processing, vol. 9784.

Atehortua, A. et al, 2016. Automatic segmentation of right ventricle in cardiac cine MR images using a saliency analysis. Med Phys, 43(12):6270.

Candemir, S. et al, 2016. Automatic heart localization and radiographic index computation in chest x-rays. Medical Imaging 2016: Computer-Aided Diagnosis, vol. 9785.

Constantinides, C. et al, 2010. Automated heart localization for the segmentation of the ventricular cavities on cine magnetic resonance images. 2010 Computing in Cardiology, 37. 911 - 914.

Fadil, H. et al, 2017. 4D Automatic Centre Detection of the Right and Left Ventricles from Cine Short-Axis MRI. Statistical Atlases and Computational Models of the Heart. Imaging and Modelling Challenges. STACOM 2016. Lecture Notes in Computer Science, vol. 10124.

Farag, A. and Fakhreldin, M., 2012. Heart Localization from Magnetic Resonance Images Sequence. Journal of Computer Science, 8(4), 499-505.

Hadhoud, M. M. A. et al, 2011. Automatic global localization of the heart from Cine MRI images. 2011 IEEE International Symposium on IT in Medicine and Education, vol.2, 35-38.

Hoffmann, R. et al, 2016. Automated Heart Localization in Cardiac Cine MR Data. Bildverarbeitung für die Medizin 2016, pp. 116-121.

Huang, J. et al, 2007. Dynamic texture based heart localization and segmentation in 4-D cardiac images. 2007 4th IEEE International Symposium on Biomedical Imaging: From Nano to Macro, 852-855.

Kim, J. et al, 2009. Automated 3D heart segmentation by search rays for building individual conductor models. Medical Imaging 2009: Visualization, Image-Guided Procedures, and Modeling, $72611 \mathrm{~W}$.

Lei, R. et al, 2016. A Novel Approach For 3D Surface Reconstruction Of LV Endocardium Based On SPCNN. 2016 4th International Conference on Electrical \& Electronics Engineering and Computer Science (ICEEECS 2016), vol. 50.

Lorenz, C. and von Berg, J., 2005. Fast automated object detection by recursive casting of search rays. International Congress Series, 1281, 230-235.

Ma, Y. et al, 2016. An SPCNN-GVF-based approach for the automatic segmentation of left ventricle in cardiac cine MR images. International Journal of Computer Assisted Radiology and Surgery, 11(11), 1951-1964.

Saalbach, A. et al, 2011. Optimizing GHT-Based Heart Localization in an Automatic Segmentation Chain. Medical Image Computing and Computer-Assisted Intervention - MICCAI 2011, 463-470.

Saleh, R. H. et al, 2016. An innovate automatic heart localization method in cardiac Cine MRI using optical flow. 2016 8th Cairo International Biomedical Engineering Conference (CIBEC), 106-109.

Sorgel, W. and Vaerman. V., 1997. Automatic heart localization from a 4D MRI data set. Proc. SPIE 3034, Medical Imaging 1997: Image Processing, vol. 3034, p. 333-344.

Tavakoli, V. and Sahba, N., 2013. Cardiac motion and strain detection using 4D CT images: comparison with tagged MRI, and echocardiography. The International Journal of Cardiovascular Imaging, 30(1), 175-184.

Zheng, Y. et al, 2008. Four-Chamber Heart Modeling and Automatic Segmentation for 3-D Cardiac CT Volumes Using Marginal Space Learning and Steerable Features. IEEE Transactions on Medical Imaging, vol. 27, no. 11, pp. 1668-1681, doi: 10.1109/TMI.2008.2004421. 